Page 1 of 24

\title{
A Multiplex CRISPR/Cas9 System for Use as an Anti-BmNPV
}

\section{Therapeutic}

Zhanqi Dong ${ }^{1 \dagger}$, Qi Qin ${ }^{1 \dagger}$, Zhigang Hu ${ }^{1}$, Peng Chen ${ }^{1}$, Liang Huang ${ }^{1}$, Jiangqiong Long ${ }^{1}$, Ting Tian ${ }^{1}$, Cheng $\mathrm{Lu}^{1,2 \ddagger}$, Minhui Pan ${ }^{1,2 \ddagger}$

State Key Laboratory of Silkworm Genome Biology, Southwest University, Chongqing 400716, China ${ }^{\mathrm{a}}$;

Key Laboratory of Sericultural Biology and Genetic Breeding, Ministry of Agriculture, Southwest University, Chongqing 400716, China ${ }^{\text {b; }}$

Running head: A multiplex CRISPR system impairs BmNPV replication

†These authors contributed equally to this work.

\#Address correspondence to Cheng Lu, lucheng@swu.edu.cn, Tel.:

+86-23-6825-0346(C.L.); Fax: +86-23-68251128 (C.L.) and Min-Hui Pan, pmh047@

126.com, Tel.:+86-23-6825-0076 (M.-H.P.); Fax: +86-23-6825-1128 (M.-H.P.).

Abstract word count: 185

Text word count: 5713 
Page 2 of 24

\begin{abstract}
:
Clustered regularly interspaced short palindromic repeats/associated protein 9 nuclease (CRISPR/Cas9) technology guided by a single-guide RNA (sgRNA) has recently opened a new avenue for antiviral therapy. A unique capability of the CRISPR/Cas9 system is multiple genome engineering. However, there are few applications in insect viruses by a single Cas 9 enzyme targeting two or more sgRNA at different genomic sites for simultaneous production of multiple DNA breaks. To address the need for multi-gene editing and sustained delivery of multiplex CRISPR/Cas9-based genome engineering tools, we developed a one-vector (pSL1180-Cas9-U6-sgRNA) system to express multiple sgRNA and Cas9 protein to excise Bombyx mori nucleopolyhedrovirus (BmNPV) in insect cells. Here, ie-1, gp64, lef-11, and dnapol genes were screened and identified as multiple sgRNA editing sites according to the BmNPV system infection and DNA replication mechanism. Furthermore, we constructed a multiplex editing vector sgMultiple to efficiently regulate multiplex gene editing steps and inhibit BmNPV replication after viral infection. This is the first report that describes the application of multiplex CRISPR/Cas9 system inhibiting insect virus replication. This multiplex system can significant enable the potential of CRISPR/Cas9-based multiplex genome engineering in transgenic silkworms.
\end{abstract}

Keyword: Bombyx mori; BmNPV; antiviral therapeutic; CRISPR/Cas9; multi-gene editing 
Page 3 of 24

\section{Introduction}

Bombyx mori nucleopolyhedrovirus (BmNPV) is an alphabaculovirus genus in the family baculoviridae $[1,2]$. BmNPV have a large circular, supercoiled, double-stranded DNA genome with 128,413 nucleotides containing 141 open reading frames (ORFs) packaged into rod-shaped virions [1,3]. Sericulture in China, India, Japan and Southeast Asia is the most developed, and BmNPV is the most common and serious disease in silkworm, accounting for about $70 \%-80 \%$ of the total losses caused by silkworm disease [4]. The main measures to prevent and control BmNPV is breeding resistant varieties via genetic breeding and transgenic breeding [5]. However, the overexpression of antiviral genes (such as Bmlispase-1, serine protease-2, Sprouty, BmAtlastin- $n$ and NADH-oxidoreductase-like protein gene) and RNA interference (RNAi) of BmNPV key genes (such as immediate early-1 (IE-1), late expression factor 1 (lef-1), lef-2, lef-3, lef-11, oral infection factor $p 74$, and gp64 gene) by transgenic technologycannot meet the needs of the sericulture industry [4-9].

An intriguing new strategy that can eradicate virus replication using the clustered regularly interspaced short palindromic repeats/CRISPR-associated protein 9 nuclease (CRISPR/Cas9) genome editing technology has been widely used in pathogenic viruses including human immunodeficiency virus 1 (HIV-1), human papillomavirus, hepatitis B virus (HBV), Epstein-Barr virus, plant virus, or flaviviruses in infected cells or animal models $[10,11]$. In the past 4 years, Cas 9 technology has been extensively utilized in basic science and transgenic breeding settings to treat genetic diseases and infectious diseases in silkworm [12-14]. Our laboratory has employed CRISPR/Cas9 technology and developed a BmNPV specific gene editing system to excise the BmNPV genome ie-1 gene from infected cells of Bombyx mori for the first time [15].

Since 2017, many of antiviral strains, including gene editing of ie- 0 , ie-1, ie-2, or me53 genes, have been cultivated through transgenic breeding mainly through strategies of signal gene editing, double gene editing, and large fragment deletion[12, 13]. At the same time, we have established a virus-inducible CRISPR/Cas9 system 
Page 4 of 24

that can reduce the off-target efficiency and host toxicity of gene editing systems. It not only could edits the key BmNPV gene directly but can also edit the virus-dependent host factors to inhibit the multiplication and replication of BmNPV [16]. All of these antiviral strains have achieved remarkable antiviral effects through transgenic genome editing. However, the introduction of nucleases into cells by these nuclease-based genome editing methods is still inefficient and has partial selectivity of viral gene editing for removing the entire BmNPV genome [13, 14]. Therefore, there is an urgent need to screen and identify BmNPV genes and used them to improve the antiviral viability by multi-gene editing in the BmNPV genome.

To achieve highly specific BmNPV genome editing, this work applied a highly efficient multiplex CRISPR/Cas9 gene editing technology to disrupt the BmNPV genome in infected silkworm cells. We first constructed a one-vector CRISPR/Cas9 gene editing system, pSL1180-Cas9-U6-sgRNA plasmid, using the BmNPV lef-11 gene as a target gene. This can quickly insert target genes and detect antiviral activity. We also screened and identified ie-1, gp64, lef-11 and dnapol genes, which are very important for viral replication based on the BmNPV systemic infection and DNA replication mechanism. More importantly, to completely eliminate the BmNPV genome from infected silkworm cells, we constructed a multiplex CRISPR/Cas9 system for using an anti-BmNPV therapeutic. The results demonstrated a series of sgRNA for highly active candidates that would be optimal for therapeutic application and successful construction of a multiplex CRISPR/Cas9 genome editing system with the ability to inhibit BmNPV replication. 
Page 5 of 24

\section{Materials and Methods}

\subsection{Cells and Viruses}

The Bombyx mori (ovarian) cell line BmN-SWU1 were grown in TC-100 medium containing penicillin/streptomycin and $10 \%$ fetal bovine serum at $27^{\circ} \mathrm{C}$ [17]. Recombinant BmNPV (v39K ${ }^{\text {prm }}$-EGFP) was constructed as previously [8, 18]. Recombinant BmNPV were generated on BmN-SWU1 cells [18].

\subsection{Plasmid construction}

The gene encoding Cas9 nuclease and the expression cassette for sgRNA were prepared as described previously [15]. The resulting Cas9 and sgRNAs expression vectors were named as pSL1180-IE1-Cas9-SV40-U6-sgRNA. Two pairs of sgRNA were designed using the CRISPR website (http://crispr.dbcls.jp/) and targeted to the amino-terminal regions of BmNPV key gene ORFs [19]. All sgRNA scaffold target sequences were inserted the $B b s$ I restriction enzyme site. All clones were verified by sequencing the plasmids. All primers used here are presented in Table S1.

\subsection{Immunofluorescence}

BmN-SWU1 cells were seeded in cover slips (Fisher Scientific, USA) in 24-well plates for $70 \%$ confluence on the day of transfections. At $48 \mathrm{~h}$ post-transfection (h p.t.), removed the cell cultures, and washed cells with PBS in three times. After transfection with the plasmids and infection with BmNPV, the BmN-SWU1 cells were studied with an immunofluorescence assay. Briefly, the samples were fixed in cold 4\% paraformaldehyde in PBS for 15 min and permeabilized with 1\% Triton X-100 for 15 min. After blocking for $1 \mathrm{~h}$ in $3 \% \mathrm{BSA}$ and $10 \%$ sheep serum in PBS at $37^{\circ} \mathrm{C}$, the cells were incubated with primary antibodies diluted 1:200 in in PBS for $1 \mathrm{~h}$. Finally, after $6 \times 5$ min washed in PBS, and incubated with Alexa 555-conjugated goat anti-mouse IgG (1:500; Life Technologies) and DAPI (1:500; Life Technologies, USA) for $1 \mathrm{~h}$, and then washed with PBS six times. Images were acquired on an OLYMPUS microscope.

\section{$2.4 \operatorname{sgRNA}$ design}

As previously reported sgRNA against BmNPV generated via the online analysis tool (http://crispr.dbcls.jp/) and the sequence listed as Table S2 [19]. Scanning for 
Page 6 of 24

$\mathrm{GN}_{19} \mathrm{NGG}$ sequences on the BmNPV genome identified anti-BmNPV sgRNA candidates that met the rules: as a target sequence and own the PAM recognition domain.

\subsection{Sequence analysis}

Wizard Genomic DNA extraction kit (Promega, USA) was used for BmNPV genome DNA extraction. The BmNPV genomic region surrounding the sgRNA target site of each gene was amplified by PCR, and detected the cleavage of the lef-11 gene. The PCR products were purified and ligated into the pEASY-T5 vector (TransGen Biotech, Beijing, China) and submitted for sequencing using universal primers (BGI, Guangzhou, China).

\subsection{Western Blot analysis}

The transfected cells were lysed in Western and IP buffer containing $20 \mathrm{mM}$ Tris-HCL, pH7.5, $150 \mathrm{mM} \mathrm{NaCl}, 1 \%$ Triton X-100 (Beyotime, China) and mixed with $5 \times$ loading buffer and boiled for $5 \mathrm{~min}$. The protein samples were separated through $12 \%$ SDS-PAGE and transferred onto the PVDF membrane. After blocking, the membranes were incubated with primary antibodies: rabbit a-VP39 (1:2000), and rabbit a-Tubulin (1:5000; Sigma, USA) in 5\% milk in TBST for $1 \mathrm{~h}$ at room temperature. After washing, the blot was treated with a HRP-labeled goat anti-rabbit $\operatorname{IgG}(1: 20,000$; Beyotime) reacted in Clarity Western ECL Substrate (Bio-Rad, USA); finally, the sample was exposed to X-ray film for detection.

\subsection{Quantitative PCR ( $q P C R)$ for DNA replication assay}

The BmNPV genome DNA was subjected to real-time PCR using SYBT Premix Ex Taq (TaKaRa, Dalian, China) following a previously described protocol and using cDNA specific primers previously by Dong et al [20]. For quantification, a DNA standard curve was constructed based on $\mathrm{Ct}$ values (Bio-Rad) as previously described for calculation of the copy number of the gp41 gene.

\subsection{Reverse transcription-PCR (RT-PCR)}

RNA extractions were performed using TRIZOL RNA extraction kit from Invitrogen research (Invitrogen, USA). The cDNA synthesis used a reverse transcription Kit (Promega). SYBR Select Master Mix Reagent (Bio-Rad, USA) was 


\section{Page 7 of 24}

using for RT-PCR reactions. The specificity of the primers was confirmed by agarose gel electrophoresis and melting curve analysis. The results are expressed as fold-increase expression of the gene of interest normalized to B. mori sw22934 gene expression using the $2^{-\Delta \Delta \mathrm{Ct}}$ method.

\subsection{Statistical analysis}

All data are presented as mean \pm SD. An unpaired, one-tailed Student's $t$ tests was used to compute the difference between two independent groups. Differences with $P<0.01$ were considered to be statistically significant. 
Page 8 of 24

\section{Results}

3.1 Establishment of a one-vector CRISPR/Cas9 system for BmNPV genome excision

BmNPV contains 141 genes most of which have not been studied [1, 21]. To systematically compare the effects of different gene knockouts on the replication of BmNPV, we constructed a one-vector CRISPR/Cas9 system for rapid detection of inhibition of BmNPV multiplication. We linked Cas9 and U6-sgRNA expression cassettes into a single pSL1180 vector, which contain two $B b s$ I digestion sites that could link the BmNPV target sequence directly (Figure 1A). Previously, we demonstrated that the BmNPV lef-11 gene is necessary for virus replication [22]. Here, we first detected gene editing and antiviral efficiency of the system by knocking out lef-11. After transfecting three different target sites for the lef-11 gene into BmN-SWU1 cells, the BmNPV genome copy was detected with the RT-PCR assay. These results showed that the amount of BmNPV DNA in the cells treated with sgLEF11-1, sgLEF11-2 and sgLEF11-3 was lower than in cells transfected with sgMock plasmids indicating that the DNA levels in the cells transfected with sgLEF11-2 was 10-fold lower (Figure 1B).

BmN-SWU1 cells were infected with the v39K ${ }^{\text {prm }}$-EGFP virus after transfecting with sgLEF-11 (sgLEF11-2), when CRISPR/Cas9 system expressed Cas9 protein, red fluorescence could be observed; otherwise, v39Kprm-EGFP virus genes expressed EGFP proteins, green fluorescence could be observed obviously. Most cells transfected with sgLEF-11 could not detected EGFP signals (Figure 1C). However, EGFP signals were detected in transfected sgMock cells (Figure 1C). Furthermore, statistical analysis showed that the number of EGFP+ cells significantly decreased from $41 \%$ to $20 \%$ after transfecting with sgLEF-11. Similarly, there was a marked reduction in the number of EGFP+/DsRed+ cells from $40 \%$ to $12 \%$ in the sgLEF-11 transfected cells (Figure 1D). Sequence analysis of the lef-11 region showed that the disruption of EGFP expression was caused by various mutations and deletions near the putative target site (Figure 1E). Most of the sequencing results showed that the deletion was about 3-12 bp at the target site. These results indicated that the 
Page 9 of 24

one-vector CRISPR/Cas9 system can rapidly identify key genes that inhibit BmNPV replication by genomic copy number and fluorescence analysis.

\section{A}

pSL1180-IE1-Cas9-SV40-U6-sgRNA

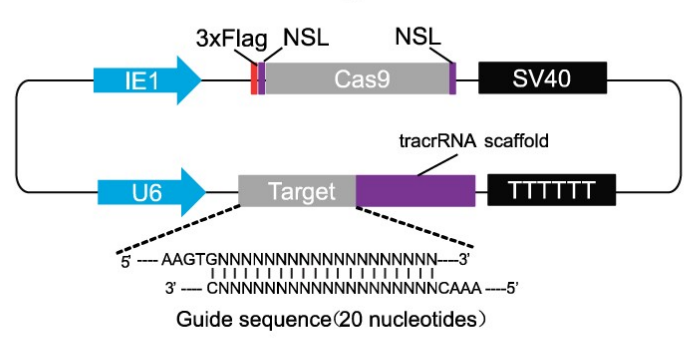

C

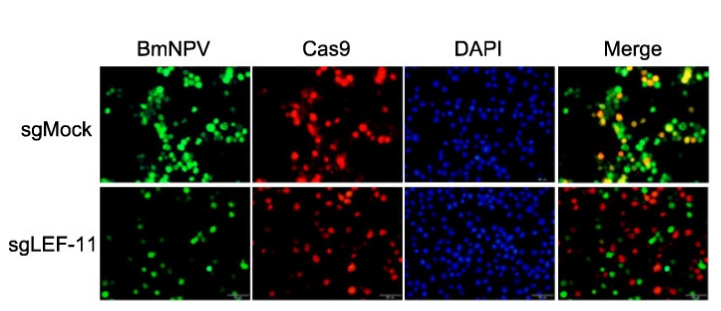

E

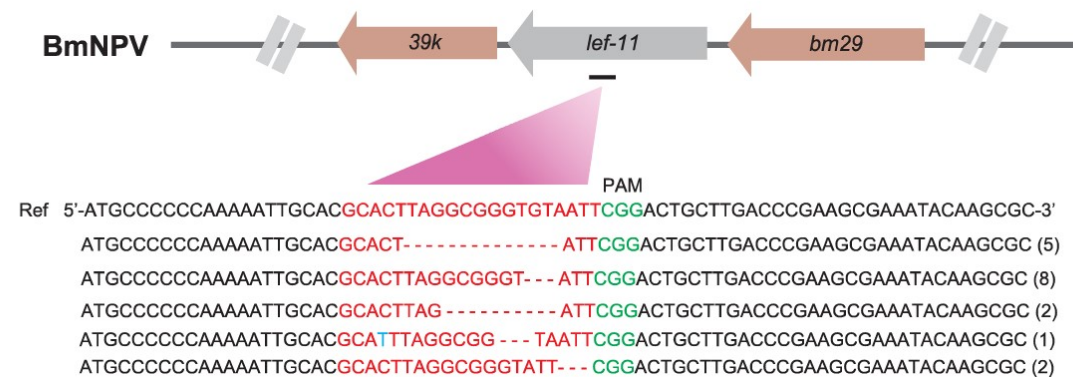

B
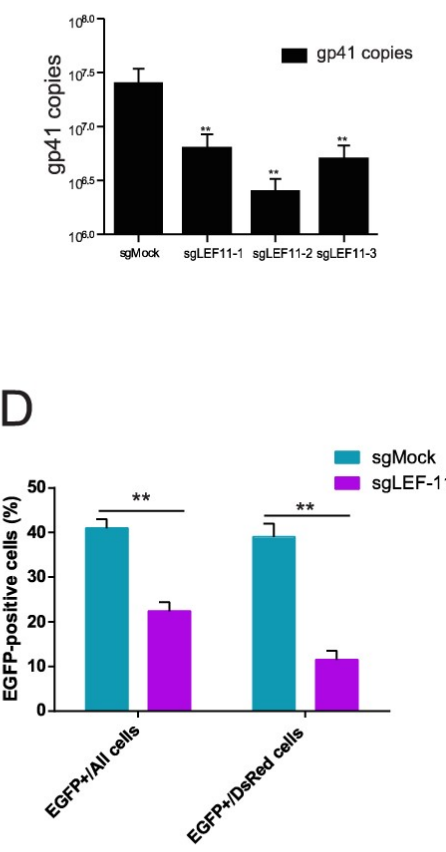
ATGCCCCCCAAAAATTGCACGCACTTAGGCGGGTATT- - - CGGACTGCTTGACCCGAAGCGAAATACAAGCGC (2)

\section{Figure 1. Establish one-vector CRISPR/Cas9 system for BmNPV genome}

excision. (A) Schematic of one vectors to sustain the CRISPR/Cas9 system. A single guide-sequence insertion site downstream of the U6 promoter was used to clone the designed sgRNA using the Bbs I restriction site. (B) DNA replication analysis of three sgLEF11 target antiviral effects. (C) Fluorescence microscopy images of BmN-SWU1 cells transfected with one-vector CRISPR/Cas9 system followed by infected with BmNPV for $72 \mathrm{~h}$ p.i.. Red represents the Cas9 protein and green represents the BmNPV infected cells. (D) BmNPV infection rates (EGFP+) in all cells and Cas9 expression cells (DsRed+) were counted by selecting five different fluorescent fields. Three independent experiments were carried out in triplicate. (E) Comparative 
Page 10 of 24

analysis for the DNA sequence of the CRISPR/Cas9 target sites in the sgLEF11 and BmNPV genome of lef-11 sequence. The WT reference sequence is shown on top in bold. The target sequence of $l e f-11$ is indicated in red. Deletions are indicated by dashes.

\subsection{Screening of key genes involved in BmNPV systemic infection}

Baculovirus replication is a cascade of regulation and is mainly divided into four periods: immediate early, early, late and very late (Figure 2A). Therefore, to reduce the screening range, we selected key genes from different periods to detect the effect on BmNPV replication after knockout using CRISPR/Cas9 system. Immediate early gene $i e-1$, is a baculovirus transcriptional activation factor, early gene gp64 affects the invasion of $\mathrm{BV}$ particles, late gene $v p 39$ is a key component of the baculovirus nucleocapsid assembly, and the very late gene poly is a key component in the formation of polyhedral virions. Thus, we selected these four genes as target genes involved in BmNPV systemic infection.

To demonstrate the antiviral efficiency of the target gene, we analyzed the amplification of the BmNPV-EGFP through fluorescence microscopy and found fewer numbers of $\mathrm{EGFP}^{+}$cells in the cells transfected with infected sgIE1 and sgGP64 than the cells transfected with sgMock (Figure 2B). However, there was a reduction in BmNPV amplification between sgVP39- or sgPOLY-transfected cells with controls sgMock (Figure 2B). Statistical analysis showed that sgIE1 had only a 23\% infection rate, sgGP64 were $26 \%$, and the controls were $51 \%$, sgVP39 or sgPOLY also had a corresponding decrease after $72 \mathrm{~h}$ p.i. (Figure 2C). The result of qPCR analysis clearly showed that the relative amount of BmNPV DNA was decreased by the cells treated with target genes but not sgMock, revealing more than a 10 -fold reduction in DNA levels in the cells transfected with sgIE1 and sgGP64 (Figure 2D). These results indicated that the excision of immediate early and very early genes from the BmNPV genome by the CRISPR/Cas9 system could effectively inhibit BmNPV replication. 
Page 11 of 24

A

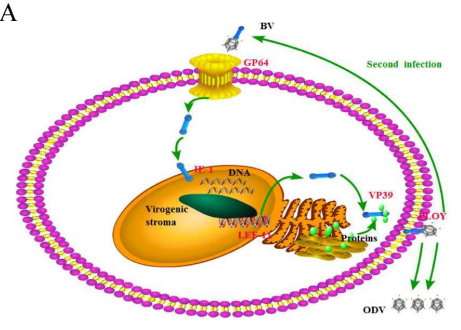

$\mathrm{C}$

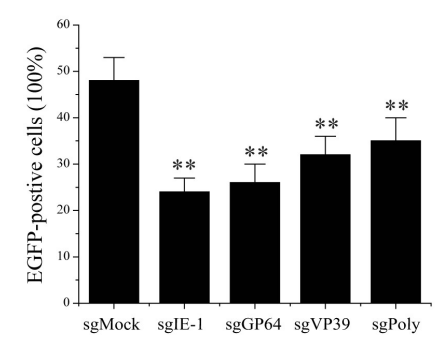

B

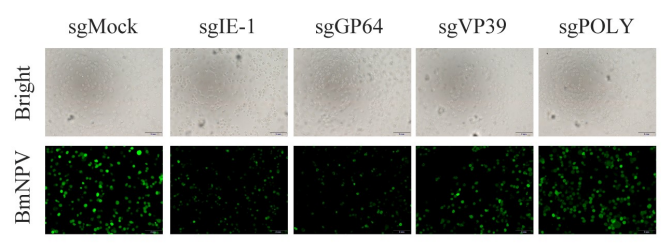

$\mathrm{D}$

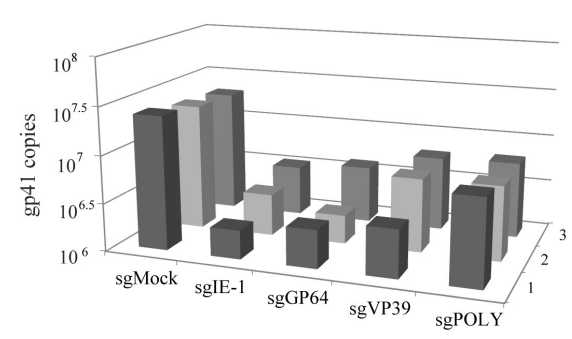

Figure 2. Screening of key gene involved in BmNPV systemic infection.

(A) Diagram of BmNPV system infection. (B) Antiviral efficiency of the target gene knockout involved in BmNPV systemic infection. BmN-SWU1 cells transfected with the indicted sgRNAs (sgMock, sgIE1, sgGP64, sgVP39 and sgPOLY) and infected with BmNPV at MOI 10 in BmN-SWU1 cells. The sgMock, sgIE1, sgGP64, sgVP39 and sgPOLY knockout cells inhibited the expression of BmNPV. Light and fluorescence images were taken $72 \mathrm{~h}$ p.i. after co-culturing the cells. (C) Statistical analysis of the EGFP-positive cells transfected with Cas9 and indicted sgRNA in the BmN-SWU1 cells. (D) BmN-SWU1 cells were transfected with indicated sgMock, sgIE1, sgGP64, sgVP39, and sgPOLY and infected with BmNPV at 10 MOI. At $72 \mathrm{~h}$ p.i., total DNA was isolated from each Cas9 transfected group and quantified by Q-PCR. Each data point was determined from the mean of three independent replicates. The sgRNA represents transfection with a target sequence for this Cas9 gene editing.

\subsection{Screening of key genes involved in BmNPV DNA replication}

The key baculovirus DNA replication genes ie-1, lef-3, p143, lef-1, and dnapol are essentially involved in DNA replication; the function of most DNA replication related genes is unknown in BmNPV (Figure 3A) [23]. According to the principle that 
Page 12 of 24

BmNPV early expression gene has a greater influence on viral DNA replication, we selected ie-1, lef-3, lef-11, ie-2, p35, p143, lef-1, and dnapol genes as the candidate target genes using a one-vector CRISPR/Cas9 system. To evaluate the efficacy of selected sgRNAs in targeting BmNPV, we transfected one-vector CRISPR/Cas9 system into BmN-SWU1 cells. At 72 h p.i., sgIE1, sgLEF-3, sgDNApol and sgLEF-11 could inhibit the expression of EGFP fluorescent protein more effectively than in the cells transfected with sgLEF1, sgP143, sgIE2, sgP35, and sgMock (Figure 3B). Statistical analysis showed that the EGFP-positive cells among transfected sgIE1, sgLEF-3, sgDNApol, and sgLEF-11 cells were only 20\%-30\% compared with 50\% for sgMock (Figure 3C). Further analysis of BmNPV DNA replication showed that sgIE1, sgDNApol and sgLEF11 were more effective at inhibiting viral DNA replication in all target genes. The number of copies of BmNPV gp4l in cells transfected with sgIE1, sgDNApol and sgLEF11 were 10-fold lower than those transfected with sgMock (Figure 3D). 
Page 13 of 24

A

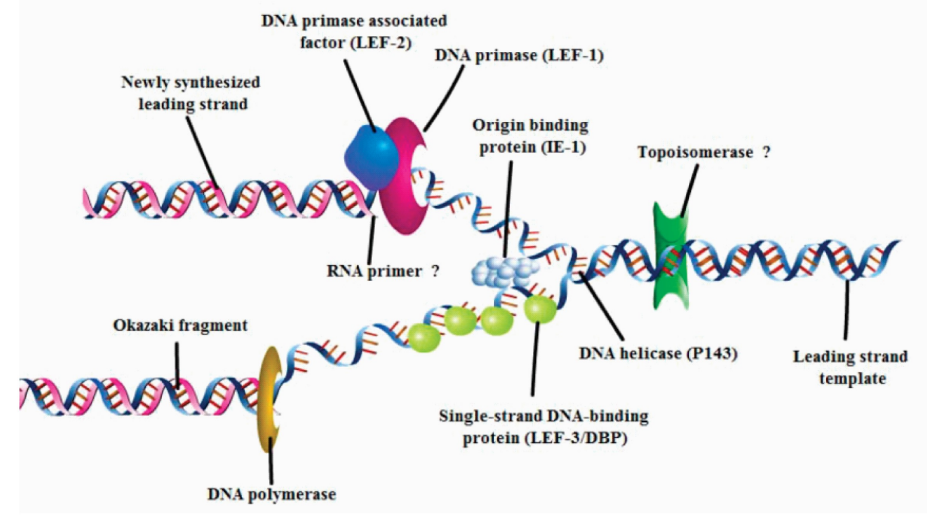

B

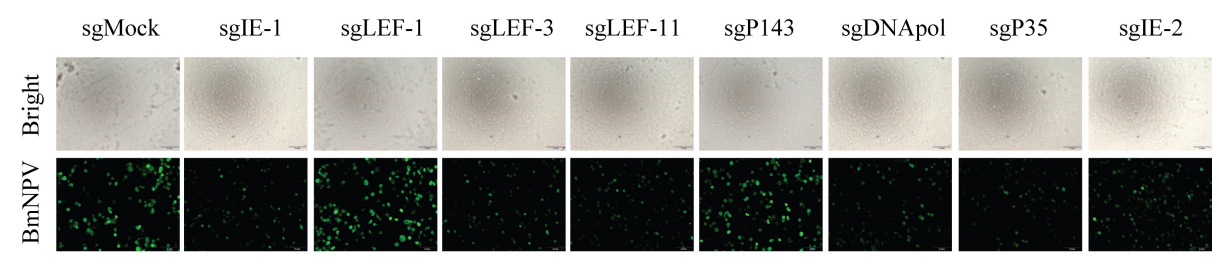

C

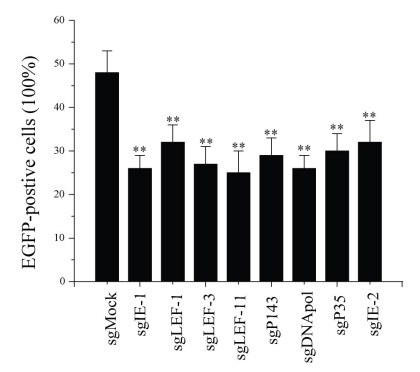

$\mathrm{D}$

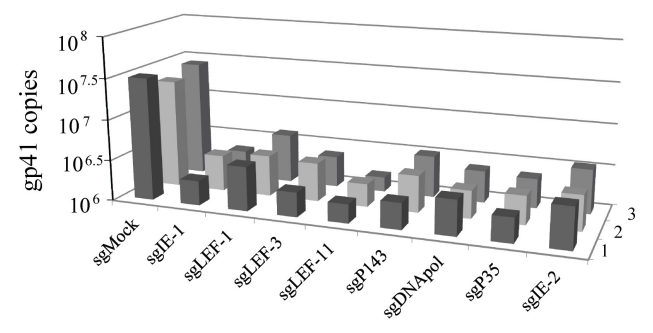

Figure 3. Screening of key genes in BmNPV DNA replication.

(A) Diagram of BmNPV DNA replication. (B) Antiviral efficiency of the target gene knockout involved in BmNPV DNA replication. BmN-SWU1 cells transfected with the indicted sgRNAs (sgMock, sgIE1, sgLEF1, sgLEF3, sgLEF11, sgP143, sgDNApol, sgP35 and sgIE2) and infected with BmNPV at MOI 10 in BmN-SWU1 cells. The sgRNA knockout cells inhibited the expression of BmNPV. Light and fluorescence images were taken $72 \mathrm{~h}$ p.i. after co-culturing the cells. (C) Statistical analysis of the EGFP-positive cell transfected with Cas9 and indicted sgRNA in the BmN-SWU1 cells. (D) BmN-SWU1 cells were transfected with indicated sgMock, sgIE1, sgLEF1, sgLEF3, sgLEF11, sgP143, sgDNApol, sgP35, and sgIE2 and infected with BmNPV at MOI 10. At $72 \mathrm{~h}$ p.i., total DNA was isolated from each Cas9 transfected group and quantified by Q-PCR. 
Page 14 of 24

\subsection{CRISPR/Cas9-mediated multiplex gene editing impairs BmNPV replication}

Next, we wondered if a combination of dual sgRNA or multiple sgRNAs could more effectively inhibit of virus replication. To examine this possibility, we used cells transduced with signal and double sgRNAs disruption of BmNPV infection via a one-vector system. The results show that the double sgRNAs inhibits viral infection more completely than targeted Cas9 (Figure S1). Thus one CRISPR/Cas9 genome editing system might be able to perform multiple sgRNAs and Cas9 gene edits simultaneously [24]. Therefore, we choose ie-1, gp64, lef-11, and dnapol as target genes for multi-gene editing through systematic screening combined with baculovirus infection process. The sgRNA and Cas9 expression cassettes were constructed into a one-vector system (Figure 4A). The vector is named PSL1180-Cas9-sgIE1-sgLEF11-sgGP64-sgDNApol (sgMultiple).

After 72 h p.i., stable expressing of sgMultiple (Red) remained uninfected with BmNPV (Green), which could observed by fluorescence microscopy, and this phenomenon shows that these cells could suppress viral replication (Figure 4B). On the contrary, there was no significant difference in sgMock compared with normal cells. Furthermore, statistical analysis showed that the average number of EGFP+ cells decreased significantly from $40 \%-45 \%$ to $18 \%-23 \%$ after transfection with sgMultiple. In addition, we compared the number of EGFP+ cells in each group of cells expressing different CRISPR/Cas9 there was a marked reduction in the number of EGFP+/DsRed+ cells from $40 \%$ to $3 \%$ in the sgMultiple transfected cells (Figure $4 C)$. We also analyzed the changes in DNA replication in different sgRNA transfected cells. At 48 h p.i, BmNPV DNA replication showed that the copy number of viral DNA decreased by $75 \%$ in the sgMultiple transfection cells. However, the DNA replication levels in sgMock were significantly higher (Figure 4D). Finally, we analyzed the effect of multiplex gene editing on the expression of BmNPV VP39 protein. These results showed that the expression of BmNPV VP39 protein in the sgMultiple transfection cells was significantly decreased compared with the control sgMock transfection cells (Figure 4E). These results suggested that the 
Page 15 of 24

CRISPR/Cas9-mediated multiplex gene editing impaired BmNPV replication and can be applied to antiviral research in transgenic silkworms.

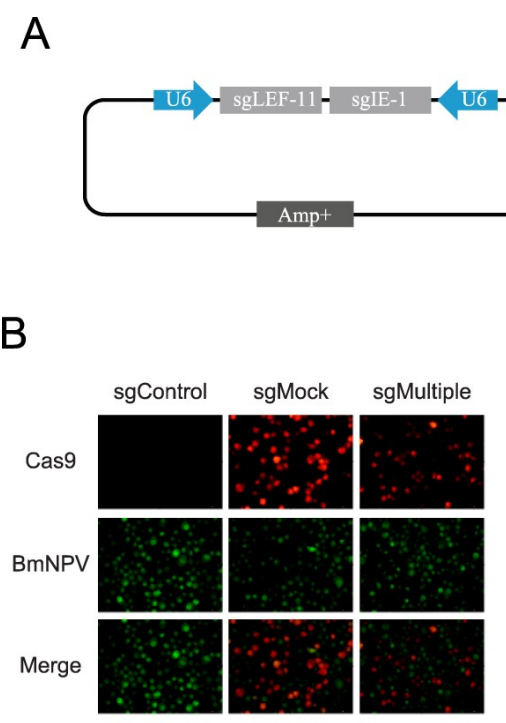

$\mathrm{D}$

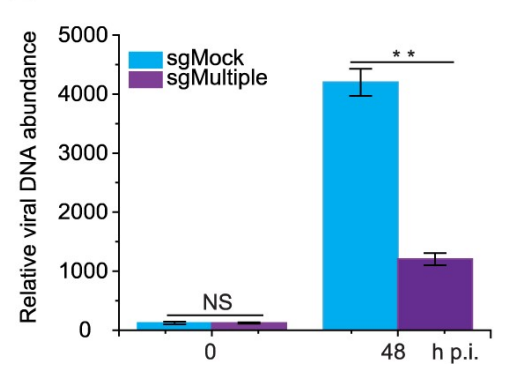

C

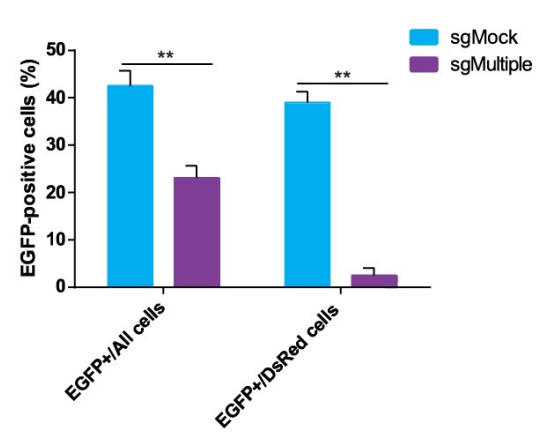

E

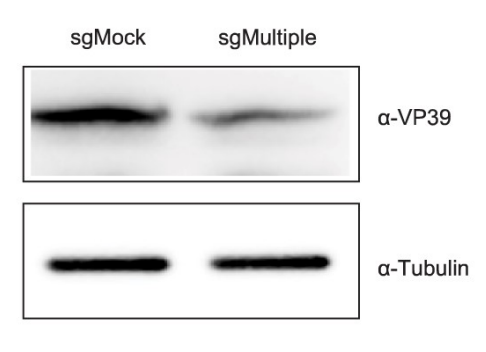

Figure 4. CRISPR/Cas9-mediated multiplex gene editing impairs BmNPV

replication. (A) Schematic of multiplex gene editing vectors to sustain the CRISPR/Cas9 system. (B) Multiplex gene editing vectors were transfected into BmN-SWU1 cells, infected with BmNPV and examined through a fluorescence microscope at $72 \mathrm{~h}$ p.i. Red represents Cas9 protein, green represents BmNPV infected cells, and DAPI represents nucleus. Besides, sgMock represents a non-target sequence and sgMultiple represents the four target sequences of this multiplex gene editing system. (C) BmNPV infection rates (EGFP-positive) in multiplex gene target cells and in non-target cells were counted by selecting five different fluorescent fields. All experiments were repeated three times. ${ }^{*}$ represent statistical significance at the level of $P<0.01$. (D) Expression of sgMultiple cells infected with BmNPV at MOI 10. 


\section{Page 16 of 24}

At $72 \mathrm{~h}$ p.i., total DNA was isolated from each Cas 9 transfected cell and quantified by Q-PCR. Each data point was determined from the mean of three independent replicates. ${ }^{* *}$ represented statistically significant differences at $P<0.01$. (E) The multiplex gene editing system mediated antiviral activity against the reporter genes as monitored by the levels of the late expression protein VP39 (top) and Tubulin (bottom), which represents the total protein expression. 
Page 17 of 24

\section{Discussion}

CRISPR/Cas9-mediated gene editing technology has been widely used in insect pest control, sex regulation, silk protein synthesis, and infectious disease research [25]. We previously we have developed a series of transgenic lines with high antiviral ability via transgenic gene editing technology $[12,13,16]$. Here, we successfully disrupted the replication of BmNPV genome utilizing the multiplex CRISPR/Cas9 system. Importantly, this disruption of BmNPV genome at multiple infection stages, blocked the transcriptional activation of viral DNA replication, as well as the invasion of BV and the synthesis of DNA polymerase. To best of our knowledge, disrupting multiple steps of viral infection with the CRISPR/Cas9 gene editing system has not yet been shown in insect cells. In future research, CRISPR-mediated multi-gene editing can be used to breed transgenic antiviral lines and insect pest control.

The Cas9 protein and sgRNA are independent after transcription, a single Cas9 protein can load multiple sgRNAs, allowing the CRISPR/Cas9 system to efficiently knock out multiple target genes [26, 27]. This has helped the genetic traits of multiple genes and the knockout of family genes [27, 28]. Multi-gene editing tools are mainly used to construct multiple gene delivery systems to deliver multiple $\operatorname{sgRNAs}$ for the treatment of HIV, retrovirus, lentivirus, and HBV [24, 29-31]. Here, we constructed a one-vector CRISPR/Cas9 system that can not only screen and identify the key genes of BmNPV systemic infection process, but also verify the antiviral efficiency of multi-gene BmNPV infection process using a multiplex CRISPR/Cas9 system (Figure 4). A critical outcome of this study is the application of multiplex systems to eliminate viral infection at multiple infection stages; it likely has value in treating transgenic silkworms.

In 2004, the BmNPV lef-1 gene was first targeted to inhibit viral replication by RNAi [6]. To date, many genes have been previously interfered with microRNA, dsRNA, shRNA, and so on in the BmNPV systemic infection process, but the target genes of these selected methods are different form our approach. Therefore, there is no systematic comparison of the antiviral efficiency of these genes [4, 8, 32]. Here, 11 genes with different functions in different stages of BmNPV infection were analyzed 
Page 18 of 24

using a one-vector CRISPR/Cas9 system (Figs. 2 and 3). The antiviral ability of different genes was systematically compared via DNA replication and viral fluorescence. These results provided many reliable target genes for the future development of transgenic antiviral silkworms. It also highlights future research directions in BmNPV gene function. Future work will combine this method to design different target genes and edit different viruses. Such as BmNPV, Bombyx mori cypovirus (BmCPV), Bombyx mori densonucleosis virus (BmDNV), and Bombyx mori flacherie virus (BmFV) [33, 34].

Other approaches will edit transgenic silkworms infected with bacterial diseases, fungal diseases and Nosema bombycis according to the characteristics of different silkworm diseases. Currently, the CRISPR family has identified gene editing systems including CRISPR/CPF1 and CRISPR/Cas13, that could edit the DNA, RNA or both DNA and RNA sequences $[35,36]$. CRISPR/CPF1 and CRISPR/Cas13 systems have smaller nuclease proteins and have more RNA target sequences and lower off-target efficiency. These can be systematically applied to research of silkworm disease resistance breeding. The results will advance the sericulture industry [37]. In the future, it will be possible to completely prevent and control silkworm infectious diseases.

In conclusion, the multiplex CRISPR/Cas9 system can use an anti-BmNPV therapeutic with great potential for genome editing. It will be applied to the field of gene therapy [38-43]. We constructed a multiplex CRISPR/Cas9 system with high efficiency gene editing and antiviral ability. The successful application of this multiplex CRISPR/Cas9 genome editing technology offers new insights to improve strategic inhibition of multiple silkworm pathogens simultaneously. 
Page 19 of 24

\section{Funding}

This work was supported by grants from the National Natural Science Foundation of China (Nos. 31472152 and 31872427), China Agriculture Research System (CARS-18), Project funded by Chongqing Special Postdoctoral Science Foundation (XmT2018020) and China Postdoctoral Science Foundation (No.80100118).

\section{Author contributions}

Data curation, Zhanqi Dong and Zhigang Hu; Formal analysis, Liang Huang; Investigation, Qi Qin and Jiangqiong Long; Software, Ting Tian; Supervision, Minhui Pan; Writing - original draft, Zhanqi Dong; Writing - review \& editing, Peng Chen, Cheng Lu and Minhui Pan.

\section{Conflicts of interest}

The authors declare no conflict of interest. 
Page 20 of 24

\section{References:}

1. Gomi, S.; Majima, K.; Maeda, S., Sequence analysis of the genome of Bombyx mori nucleopolyhedrovirus. J Gen Virol 1999, 80 ( Pt 5), 1323-37.

2. Kok, I. P.; Skuratovskaia, I. N., [Circular replicating baculovirus genome]. Tsitol Genet 1988, 22, (5), 39-43.

3. VS, M. I., [Replication of the baculovirus genome]. Mol Biol (Mosk) 2003, 37, (2), 288-99.

4. Jiang, L.; Xia, Q., The progress and future of enhancing antiviral capacity by transgenic technology in the silkworm Bombyx mori. Insect Biochem Mol Biol 2014, 48, 1-7.

5. Ponnuvel, K. M.; Nakazawa, H.; Furukawa, S.; Asaoka, A.; Ishibashi, J.; Tanaka, H.; Yamakawa, M., A lipase isolated from the silkworm Bombyx mori shows antiviral activity against nucleopolyhedrovirus. J Virol 2003, 77, (19), 10725-9.

6. Isobe, R.; Kojima, K.; Matsuyama, T.; Quan, G. X.; Kanda, T.; Tamura, T.; Sahara, K.; Asano, S. I.; Bando, H., Use of RNAi technology to confer enhanced resistance to BmNPV on transgenic silkworms. Arch Virol 2004, 149, (10), 1931-40.

7. Zhang, P.; Wang, J.; Lu, Y.; Hu, Y.; Xue, R.; Cao, G.; Gong, C., Resistance of transgenic silkworm to BmNPV could be improved by silencing ie-1 and lef-1 genes. Gene Ther 2014, 21, (1), 81-8.

8. Zhang, J.; He, Q.; Zhang, C. D.; Chen, X. Y.; Chen, X. M.; Dong, Z. Q.; Li, N.; Kuang, X. X.; Cao, M. Y.; Lu, C.; Pan, M. H., Inhibition of BmNPV replication in silkworm cells using inducible and regulated artificial microRNA precursors targeting the essential viral gene lef-11. Antiviral Res 2014, 104, 143-52.

9. Yao, H.; He, F.; Guo, A.; Cao, C.; Lu, X.; Wu, X., Gene analysis of an antiviral protein SP-2 from Chinese wild silkworm, Bombyx mandarina Moore and its bioactivity assay. Sci China C Life Sci 2008, 51, (10), 879-84.

10. Lijuan, Y.; Siqi, H.; Fei, G., [The application of CRISPR-Cas9 gene editing technology in viral infection diseases]. Yi Chuan 2015, 37, (5), 412-8.

11. Pellagatti, A.; Dolatshad, H.; Valletta, S.; Boultwood, J., Application of CRISPR/Cas9 genome editing to the study and treatment of disease. Archives of toxicology 2015, 89, (7), 1023-34.

12. Dong, Z.; Dong, F.; Yu, X.; Huang, L.; Jiang, Y.; Hu, Z.; Chen, P.; Lu, C.; Pan, M., Excision of Nucleopolyhedrovirus Form Transgenic Silkworm Using the CRISPR/Cas9 System. Front Microbiol 2018, 9, 209.

13. Dong, Z.; Hu, Z.; Qin, Q.; Dong, F.; Huang, L.; Long, J.; Chen, P.; Lu, C.; 
Page 21 of 24

Pan, M., CRISPR/Cas9 Mediated Disruption of the ie-0 and ie-2 as a Therapeutic Approach to BmNPV in Transgenic Silkworm. Insect Mol Biol 2018.

14. Chen, S.; Hou, C.; Bi, H.; Wang, Y.; Xu, J.; Li, M.; James, A. A.; Huang, Y.; Tan, A., Transgenic Clustered Regularly Interspaced Short Palindromic Repeat/Cas9-Mediated Viral Gene Targeting for Antiviral Therapy of Bombyx mori Nucleopolyhedrovirus. J Virol 2017, 91, (8).

15. Dong, Z. Q.; Chen, T. T.; Zhang, J.; Hu, N.; Cao, M. Y.; Dong, F. F.; Jiang, Y. M.; Chen, P.; Lu, C.; Pan, M. H., Establishment of a highly efficient virus-inducible CRISPR/Cas9 system in insect cells. Antiviral Res 2016, 130, 50-7.

16. Dong, Z.; Huang, L.; Dong, F.; Hu, Z.; Qin, Q.; Long, J.; Cao, M.; Chen, P.; Lu, C.; Pan, M. H., Establishment of a baculovirus-inducible CRISPR/Cas9 system for antiviral research in transgenic silkworms. Appl Microbiol Biotechnol 2018.

17. Pan, M. H.; Cai, X. J.; Liu, M.; Lv, J.; Tang, H.; Tan, J.; Lu, C., Establishment and characterization of an ovarian cell line of the silkworm, Bombyx mori. Tissue Cell 2010, 42, (1), 42-6.

18. Dong, Z. Q.; Zhang, J.; Chen, X. M.; He, Q.; Cao, M. Y.; Wang, L.; Li, H. Q.; Xiao, W. F.; Pan, C. X.; Lu, C.; Pan, M. H., Bombyx mori nucleopolyhedrovirus ORF79 is a per os infectivity factor associated with the PIF complex. Virus Res 2014, 184, 62-70.

19. Naito, Y.; Hino, K.; Bono, H.; Ui-Tei, K., CRISPRdirect: software for designing CRISPR/Cas guide RNA with reduced off-target sites. Bioinformatics 2015, 31, (7), 1120-3.

20. Dong, Z. Q.; Hu, N.; Dong, F. F.; Chen, T. T.; Jiang, Y. M.; Chen, P.; Lu, C.; Pan, M. H., Baculovirus LEF-11 Hijack Host ATPase ATAD3A to Promote Virus Multiplication in Bombyx mori cells. Sci Rep 2017, 7, 46187.

21. Ono, C.; Kamagata, T.; Taka, H.; Sahara, K.; Asano, S.; Bando, H., Phenotypic grouping of $141 \mathrm{BmNPVs}$ lacking viral gene sequences. Virus Res 2012, 165, (2), 197-206.

22. Dong, Z. Q.; Hu, N.; Zhang, J.; Chen, T. T.; Cao, M. Y.; Li, H. Q.; Lei, X. J.; Chen, P.; Lu, C.; Pan, M. H., Oligomerization of Baculovirus LEF-11 Is Involved in Viral DNA Replication. PLoS One 2015, 10, (12), e0144930.

23. Vanarsdall, A. L.; Mikhailov, V. S.; Rohrmann, G. F., Baculovirus DNA replication and processing. Curr Drug Targets 2007, 8, (10), 1096-102.

24. Schiwon, M.; Ehrke-Schulz, E.; Oswald, A.; Bergmann, T.; Michler, T.; Protzer, U.; Ehrhardt, A., One-Vector System for Multiplexed CRISPR/Cas9 
Page 22 of 24

against Hepatitis B Virus cccDNA Utilizing High-Capacity Adenoviral Vectors. Mol Ther Nucleic Acids 2018, 12, 242-253.

25. Ma, S. Y.; Smagghe, G.; Xia, Q. Y., Genome editing in Bombyx mori: New opportunities for silkworm functional genomics and the sericulture industry. Insect Sci 2018.

26. Zhang, J.; Graham, S.; Tello, A.; Liu, H.; White, M. F., Multiple nucleic acid cleavage modes in divergent type III CRISPR systems. Nucleic Acids Res 2016, 44, (4), 1789-99.

27. Jang, D. E.; Lee, J. Y.; Lee, J. H.; Koo, O. J.; Bae, H. S.; Jung, M. H.; Bae, J. H.; Hwang, W. S.; Chang, Y. J.; Lee, Y. H.; Lee, H. W.; Yeom, S. C., Multiple sgRNAs with overlapping sequences enhance CRISPR/Cas9-mediated knock-in efficiency. Exp Mol Med 2018, 50, (4), 16.

28. Schiml, S.; Puchta, H., Revolutionizing plant biology: multiple ways of genome engineering by CRISPR/Cas. Plant Methods 2016, 12, 8.

29. Yin, L.; Hu, S.; Mei, S.; Sun, H.; Xu, F.; Li, J.; Zhu, W.; Liu, X.; Zhao, F.; Zhang, D.; Cen, S.; Liang, C.; Guo, F., CRISPR/Cas9 Inhibits Multiple Steps of HIV-1 Infection. Hum Gene Ther 2018.

30. Hu, Y.; O'Boyle, K.; Auer, J.; Raju, S.; You, F.; Wang, P.; Fikrig, E.; Sutton, R. E., Multiple UBXN family members inhibit retrovirus and lentivirus production and canonical NFkappaBeta signaling by stabilizing IkappaBalpha. PLoS Pathog 2017, 13, (2), e1006187.

31. Sakuma, T.; Masaki, K.; Abe-Chayama, H.; Mochida, K.; Yamamoto, T.; Chayama, K., Highly multiplexed CRISPR-Cas9-nuclease and Cas9-nickase vectors for inactivation of hepatitis B virus. Genes Cells 2016, 21, (11), $1253-1262$.

32. Jiang, L.; Zhao, P.; Wang, G.; Cheng, T.; Yang, Q.; Jin, S.; Lin, P.; Xiao, Y.; Sun, Q.; Xia, Q., Comparison of factors that may affect the inhibitory efficacy of transgenic RNAi targeting of baculoviral genes in silkworm, Bombyx mori. Antiviral Res 2013, 97, (3), 255-63.

33. Cao, G.; Meng, X.; Xue, R.; Zhu, Y.; Zhang, X.; Pan, Z.; Zheng, X.; Gong, C., Characterization of the complete genome segments from BmCPV-SZ, a novel Bombyx mori cypovirus 1 isolate. Can J Microbiol 2012, 58, (7), 872-83.

34. Li, Y.; Zadori, Z.; Bando, H.; Dubuc, R.; Fediere, G.; Szelei, J.; Tijssen, P., Genome organization of the densovirus from Bombyx mori (BmDNV-1) and enzyme activity of its capsid. J Gen Virol 2001, 82, (Pt 11), 2821-5.

35. Tsukamoto, T.; Sakai, E.; Iizuka, S.; Taracena-Gandara, M.; Sakurai, F.; Mizuguchi, H., Generation of the Adenovirus Vector-Mediated CRISPR/Cpf1 System and the Application for Primary Human Hepatocytes Prepared from 
Page 23 of 24

Humanized Mice with Chimeric Liver. Biol Pharm Bull 2018, 41, (7), 1089-1095.

36. Mahas, A.; Mahfouz, M., Engineering virus resistance via CRISPR-Cas systems. Curr Opin Virol 2018, 32, 1-8.

37. Konermann, S.; Lotfy, P.; Brideau, N. J.; Oki, J.; Shokhirev, M. N.; Hsu, P. D., Transcriptome Engineering with RNA-Targeting Type VI-D CRISPR Effectors. Cell 2018, 173, (3), 665-676 e14.

38. Li, J. F.; Zhang, D.; Sheen, J., Targeted plant genome editing via the CRISPR/Cas9 technology. Methods in molecular biology 2015, 1284, 239-55.

39. Li, K.; Wang, G.; Andersen, T.; Zhou, P.; Pu, W. T., Optimization of genome engineering approaches with the CRISPR/Cas9 system. PloS one 2014, 9, (8), e105779.

40. Li, L.; He, Z. Y.; Wei, X. W.; Gao, G. P.; Wei, Y. Q., Challenges in CRISPR/CAS9 Delivery: Potential Roles of Nonviral Vectors. Human gene therapy 2015, 26, (7), 452-62.

41. Riordan, S. M.; Heruth, D. P.; Zhang, L. Q.; Ye, S. Q., Application of CRISPR/Cas9 for biomedical discoveries. Cell \& bioscience 2015, 5, 33.

42. Savic, N.; Schwank, G., Advances in therapeutic CRISPR/Cas9 genome editing. Translational research : the journal of laboratory and clinical medicine 2015.

43. Asensio, C. S., [Application of Cas9/CRISPR to the study of synaptic function]. Medecine sciences : M/S 2015, 31, (2), 137-8. 
Page 24 of 24

Supporting information

Figure S1.

Comparison of CRISPR/Cas9 editing efficiency of single and double sgRNAs. (A) BmN-SWU1 cells transfected with the indicted sgMock, signal sgRNAs (sgIE1, sgGP64, sgLEF11), or double sgRNAs (sgLEF11\&IE1 sgLEF11\&GP64, sgIE1\&GP64) and infected with BmNPV at MOI 10 in BmN-SWU1 cells. The sgRNA knockout cells inhibited the expression of BmNPV. Light and fluorescence images were taken $96 \mathrm{~h}$ p.i. after co-culturing the cells. (B) Statistical analysis of the EGFP-positive cells transfected with Cas9 and indicted sgRNA in the BmN-SWU1 cells.

Table S1. Sequences of primers used in this study.

Table S2. The sgRNA target sequences of primers used in this study. 